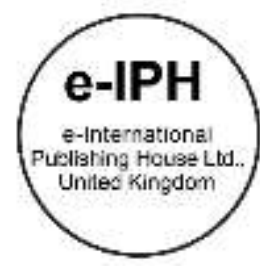

\title{
Wheelchair Skills Training: A scoping review
}

\author{
Mohd Zulkifli Abdul Rahim¹, Nurul Akrami Ab Rahim Yang², Ahmad Zamir Che Daud³ \\ 1 Disability Transformation Unit, School of Health Sciences, Universiti Sains Malaysia,Malaysia, ${ }^{2}$ Faculty of Health Sciences, Universiti \\ Teknologi MARA, ${ }^{3}$ Centre of Occupational Therapy, Faculty of Health Sciences, Universiti Teknologi MARA \\ mdzul@usm.my, nurulakrami94@gmail.com, zamir5853@uitm.edu.my \\ Tel of 1st Author: 0139301361
}

\begin{abstract}
This study aimed to identify the types of available evidence and analyze knowledge gaps on wheelchair skills training among PWDs in literature. This study employed a scoping review method and was guided by Arksey and O'Malley's using five steps framework. A total of 15 peer-reviewed articles met the inclusion criteria. The results support the effectiveness of wheelchair skills training through personal feedbacks from participants, observation and the use of standardized outcome measures, including self-perceived assessment. This study highlights the importance and benefit of wheelchair skills training to wheelchair users, caregivers and health personnel.
\end{abstract}

Keywords: Keywords: Skill Training; Disability; Wheelchair; Scoping Review

eISSN: 2398-4287@ 2021. The Authors. Published for AMER ABRA cE-Bs by e-International Publishing House, Ltd., UK. This is an open access article under the CC BYNC-ND license (http://creativecommons.org/licenses/by-nc-nd/4.0). Peer-review under responsibility of AMER (Association of Malaysian Environment-Behaviour Researchers), ABRA (Association of Behavioural Researchers on Asians/Africans/Arabians) and cE-Bs (Centre for Environment-Behaviour Studies), Faculty of Architecture, Planning \& Surveying, Universiti Teknologi MARA, Malaysia.

DOI: https://doi.org/10.21834/ebpj.v6i17.2879

\subsection{Introduction}

World Health Organization (2012) defines a wheelchair as a device that provides wheeled mobility and seating support for a person who has difficulty in walking or moving around. Wheelchair serves as a primary means of mobility for persons with physical disabilities. A fit wheelchair is a wheelchair that meets the users' needs and environmental conditions. Wheelchairs have been shown to improve the lives of people with disabilities by enabling independent mobility, employment, and equal participation in community life (Sumner, O'Connell, \& Macalpine, 2017). Wheelchairs have been proved to increase wheelchair users' participation in community and employment (Sumner et al., 2017).

Despite the proven positive impact on the life of wheelchair users, they also experienced secondary injuries as a result from long term used of a wheelchair, such as a shoulder injury (Leving, et al 2018; Morgan et al, 2017; Boninger et al 2005), pressure sores (Desai et al , 2018;Toro, et al, 2016), scoliosis (Walsh, 2009), muscular injuries (Lemay,Routhier, Noreau, Phang \& Ginis, 2012), fatigue and pain in hip the joint ( Desai et al., 2018), postural deformities as well as injuries as a results of falling (Forslund et al, 2017;Best, Routhier, \& Miller, 2015;Rice, Ousley and Sosnoff, 2015; Xiang,Chany \& Smith, 2006).

Morgan et al., (2015) state that wheelchair skills may reduce wheelchair users' injuries and help them maneuver independently. Previous studies have stated that wheelchair skills are one of the factors affecting social and community participation in wheelchair users (Smith, 2014). Wheelchair skills training has been shown to prevent future health and participation restrictions (Morgan et al., 2015), improves wheelchair users' self-efficacy (Best et al., 2015) and increase confidence among inexperienced wheelchair users (Sakakibara et al., 2013).

eISSN: 2398-4287C 2021. The Authors. Published for AMER ABRA cE-Bs by e-International Publishing House, Ltd., UK. This is an open access article under the CC BYNC-ND license (http://creativecommons.org/licenses/by-nc-nd/4.0/). Peer-review under responsibility of AMER (Association of Malaysian Environment-Behaviour Researchers), ABRA (Association of Behavioural Researchers on Asians/Africans/Arabians) and cE-Bs (Centre for Environment-Behaviour Studies), Faculty of Architecture, Planning \& Surveying, Universiti Teknologi MARA, Malaysia.

DOI: https://doi.org/10.21834/ebpj.v6i17.2879 
Wheelchair skills training is used by trainers and health personnels to help wheelchair users to improve their skills in using wheelchair and improve wheelchair handling skills for caregivers. To assist wheelchair users and caregivers, the wheelchair research team at the Dalhousie University and the Nova Scotia Rehabilitation Centre has introduced a Wheelchair Skills Program (WSP). The WSP is a set of skills that wheelchair users and caregivers need to perform regularly and had been widely used to train wheelchair users and caregivers (Kirby et al, 2019). However, formal wheelchair skills training is still relatively uncommon in Malaysia. Therefore, this scoping review was aimed to examine the types of available evidence and knowledge gaps on wheelchair skills training among PWDs in literature.

\subsection{Research Method}

This research will be using scoping review using Arksey and O'Malley methodological framework. Scoping review is used to describe existing literature and other sources of information and usually include such study findings from different study design and methods (Sucharew \& Macaluso, 2019). The methodological framework of scoping review by Arksey and O'Malley (2005) introduces five steps to guide researchers; identifying the research question, identifying relevant studies, study selection, charting the data, and collating, summarizing, and reporting results. Arksey and O'Malley's (2005) five steps are outlined as follows;

i) Identifying the Research Question

The research question of this study is to examine the types of available evidence and knowledge gaps on wheelchair skills training among PWDs in literature.

ii) Identifying Relevant Studies

The following databases were searched: EBSCOhost, ProQuest, ScienceDirect, Web of Science and google scholar. Keywords used are wheelchair skills, wheelchair training, wheelchair skills training, wheelchair skills program, wheelchair skill test, wheelchair skill test questionnaire and wheelchair program.
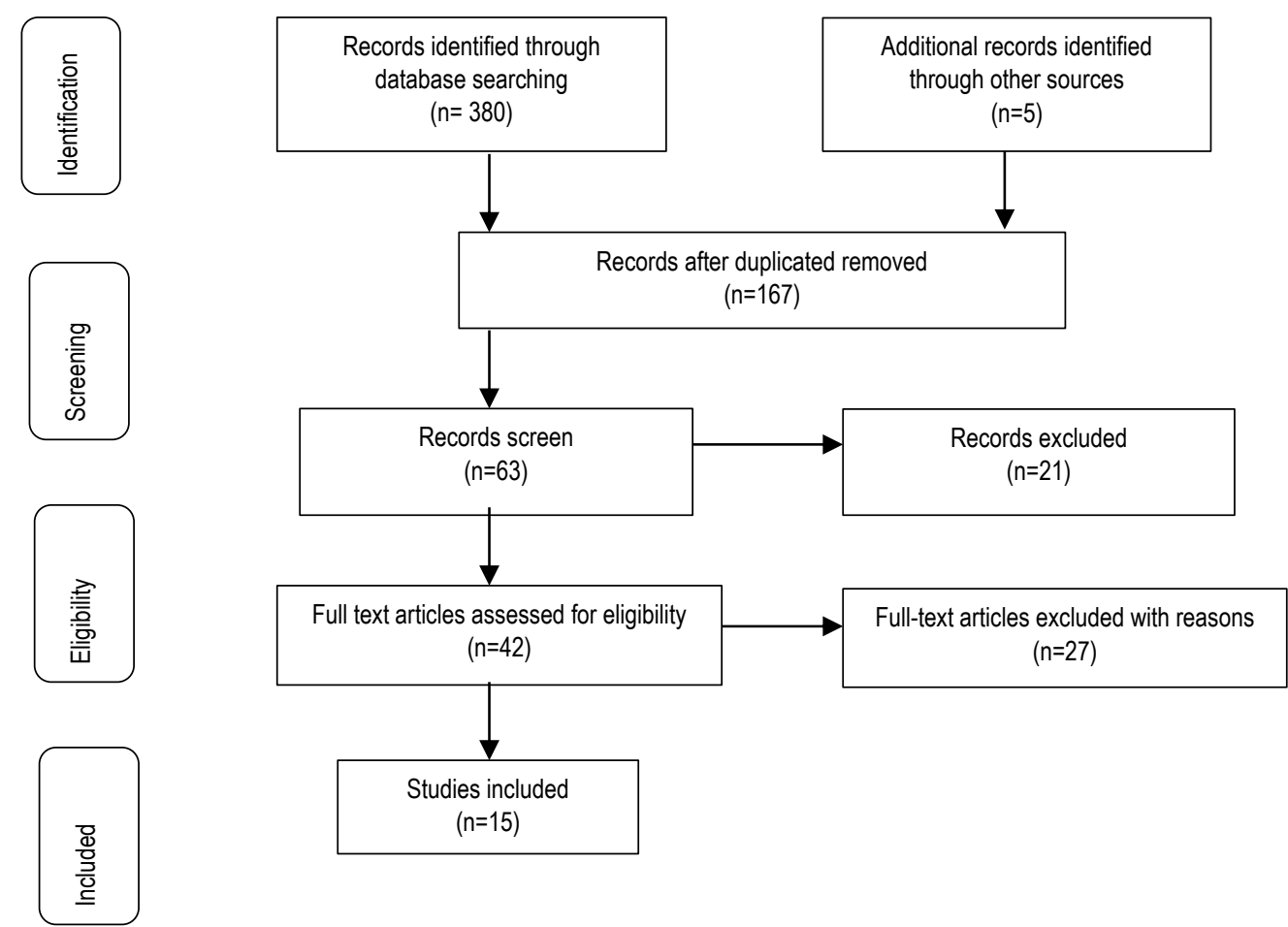

Fig 1 Flow Diagram for Scoping Review

\section{iii) Study Selection}

Studies were selected using the inclusion and exclusion criteria. The inclusion criteria are the journals must be published from 2000 to 2020, written in English and are peer reviewed. The journals were not included if the journals were published before 2000, not written in English and are books or from chapters.

iv) Data Charting

Three reviewers screened the articles to extract relevant studies based on the inclusion and exclusion criteria. A table was created to chart the relevant data and to critically appraise the papers. The categories are the citation, objectives of research, study design, sample size, intervention or module, outcome measures and findings. The information is recorded in table 1.1.

v) Collating, Summarizing, And Reporting Results.

The results and findings of the scoping review was discussed under themes that highlight the efficacy of wheelchair skills training. 
Table 1.1: The summarization of journal in wheelchair skills training

\begin{tabular}{|c|c|c|c|c|c|c|}
\hline Author & Aim & Study Design & Sample size & $\begin{array}{l}\text { Intervention/ } \\
\text { Module }\end{array}$ & $\begin{array}{l}\text { Outcomes } \\
\text { measure }\end{array}$ & Findings \\
\hline $\begin{array}{l}\text { Coolen, } \\
\text { Kirby, } \\
\text { MacPhee, } \\
\text { Dupuis, } \\
\text { Smith, Best, } \\
\text { MacKenzie } \\
\text { \& MacLeod } \\
\text { (2004) }\end{array}$ & $\begin{array}{l}\text { - To test the hypothesis that a } \\
\text { brief formalized period of } \\
\text { wheelchair skills training, } \\
\text { added to the standard } \\
\text { curriculum, cause } \\
\text { significantly greater overall } \\
\text { improvements in wheelchair } \\
\text { skills than a standard } \\
\text { undergraduate occupational } \\
\text { therapy (OT) curriculum } \\
\text { alone. }\end{array}$ & $\begin{array}{l}- \text { Randomized } \\
\text { controlled trial. }\end{array}$ & $\begin{array}{l}\text { - } 82 \text { students in a } \\
\text { university } \\
\text { undergraduate } \\
\text { OT program. }\end{array}$ & $\begin{array}{l}\text { - Using WSTP } \\
\text { module } \\
\text { - For intervention } \\
\text { group, students } \\
\text { are allocated to } \\
\text { the Wheelchair } \\
\text { Skills Training } \\
\text { Program (WSTP) } \\
\text { group, were } \\
\text { trained (on a } \\
\text { single occasion } \\
\text { each, in groups of } \\
\text { 1-3 at a time) on } \\
\text { the } 50 \text { skills that } \\
\text { make up the } \\
\text { WSTP. } \\
\text { - Students in } \\
\text { control group only } \\
\text { receive standard } \\
\text { university } \\
\text { curriculum. }\end{array}$ & $\begin{array}{l}\text { Wheelchair } \\
\text { Skills Test } \\
\text { (WST), Version } \\
2.4 \text {. }\end{array}$ & $\begin{array}{l}\text { - The WSTP is an } \\
\text { effective way to } \\
\text { improve the } \\
\text { wheelchair-skills } \\
\text { performance of } \\
\text { OT students. } \\
\text { - WSTP group } \\
\text { score higher in } \\
\text { mean } \\
\text { percentage of } \\
\text { WST. }\end{array}$ \\
\hline $\begin{array}{l}\text { MacPhee, } \\
\text { Kirby, } \\
\text { Coolen, } \\
\text { Smith, } \\
\text { MacLeod \& } \\
\text { Dupuis } \\
\text { (2004) }\end{array}$ & $\begin{array}{l}\text { - To test the hypothesis that a } \\
\text { brief, formalized period of } \\
\text { additional wheelchair skills } \\
\text { training is safe and results in } \\
\text { significantly greater } \\
\text { improvements in wheelchair } \\
\text { skills performance than a } \\
\text { standard rehabilitation } \\
\text { program. }\end{array}$ & $\begin{array}{l}\text { - Randomized } \\
\text { controlled trial. }\end{array}$ & $\begin{array}{l}\text { - } 35 \text { wheelchair } \\
\text { users admitted } \\
\text { for initial } \\
\text { rehabilitation. }\end{array}$ & $\begin{array}{l}\text { - Using WSP } \\
\text { Module. } \\
\text { - Subjects randomly } \\
\text { allocated to the } \\
\text { treatment group } \\
\text { participated in the } \\
\text { Wheelchair Skills } \\
\text { Training Program } \\
\text { (WSTP), } \\
\text { averaging 4.5-1.5 } \\
\text { training sessions, } \\
\text { each } 30 \text { minutes } \\
\text { long. }\end{array}$ & $\begin{array}{l}\text { - Wheelchair } \\
\text { Skills Test } \\
\text { (WST), version } \\
2.4 \text {. }\end{array}$ & $\begin{array}{l}\text { - The WSTP is } \\
\text { safe and } \\
\text { practical and } \\
\text { has a clinically } \\
\text { significant effect } \\
\text { on the } \\
\text { independent } \\
\text { wheeled } \\
\text { mobility of new } \\
\text { wheelchair } \\
\text { users. } \\
\text { - WSTP groups } \\
\text { shows higher } \\
\text { improvements. }\end{array}$ \\
\hline $\begin{array}{l}\text { Best, Kirby, } \\
\text { Smith, } \\
\text { MacLeod } \\
(2005)\end{array}$ & $\begin{array}{l}\text { - To test the hypotheses that } \\
\text { wheelchair skills training of } \\
\text { community-based manual } \\
\text { wheelchair users is } \\
\text { efficacious, safe, and } \\
\text { practical. }\end{array}$ & $\begin{array}{l}\text { - A randomized } \\
\text { controlled trial. }\end{array}$ & $\begin{array}{l}\text { - } 20 \text { community- } \\
\text { based manual } \\
\text { wheelchair } \\
\text { uses. }\end{array}$ & $\begin{array}{l}\text { - Using Wheelchair } \\
\text { Skills Training } \\
\text { Program (WSTP). } \\
\text { - Participants } \\
\text { allocated to } \\
\text { WSTP or control } \\
\text { group. } \\
\text { - In WSTP group, } \\
\text { participants } \\
\text { receive a mean of } \\
45 \text { hours of } \\
\text { training. }\end{array}$ & $\begin{array}{l}\text { - Wheelchair } \\
\text { Skills Test } \\
\text { (WST, version } \\
\text { 3.1) }\end{array}$ & $\begin{array}{l}\text { - Wheelchair } \\
\text { skills training of } \\
\text { community- } \\
\text { based manual } \\
\text { wheelchair } \\
\text { users is } \\
\text { efficacious, } \\
\text { safe, and } \\
\text { practical. } \\
\text { - WSTP group } \\
\text { have } \\
\text { significantly } \\
\text { higher scores in } \\
\text { post-test. }\end{array}$ \\
\hline $\begin{array}{l}\text { Kirby, } \\
\text { Smith, } \\
\text { Billard, } \\
\text { Irving, Pitts } \\
\text { \& White } \\
(2010)\end{array}$ & $\begin{array}{l}\text { - To examine the hypotheses } \\
\text { that training a parent in } \\
\text { wheelchair-user and } \\
\text { caregiver wheelchair skills } \\
\text { would improve the child's } \\
\text { wheelchair skills }\end{array}$ & - Case study & $\begin{array}{l}\text { - An 11-year-old } \\
\text { girl with spina } \\
\text { bifida and her } \\
\text { mother. }\end{array}$ & $\begin{array}{l}\text { - WSTP } \\
\text { - The mother } \\
\text { received } 4 \text { training } \\
\text { sessions } \\
\text { averaging } 42.5 \\
\text { minutes per } \\
\text { session over a } \\
\text { period of three } \\
\text { weeks. }\end{array}$ & $\begin{array}{l}\text { - Wheelchair } \\
\text { Skills Test } \\
\text { (WST 4.1) }\end{array}$ & $\begin{array}{l}\text { - The WST score } \\
\text { of the child and } \\
\text { mother shows } \\
\text { improvements. } \\
\text { - WST scores for } \\
\text { mother is higher } \\
\text { after training } \\
\text { than the child. }\end{array}$ \\
\hline $\begin{array}{l}\text { Routhier, } \\
\text { Kirby, } \\
\text { Demers, } \\
\text { Depa \& } \\
\text { Thompson } \\
(2012)\end{array}$ & $\begin{array}{l}\text { - To test the hypotheses that } \\
\text { wheelchair users who } \\
\text { received the French- } \\
\text { Canadian version of the } \\
\text { Wheelchair Skills Training } \\
\text { (WSTP) would significantly } \\
\text { improves their wheelchair- } \\
\text { skills capacity and that these } \\
\text { improvements would be } \\
\text { retained at three months }\end{array}$ & $\begin{array}{l}\text { - Multicenter, } \\
\text { single-blind, } \\
\text { randomized } \\
\text { controlled trial. }\end{array}$ & $\begin{array}{l}\text { - } 39 \text { manual } \\
\text { wheelchair } \\
\text { users. }\end{array}$ & $\begin{array}{l}\text { - Participants in } \\
\text { WSTP group } \\
\text { received standard } \\
\text { care with a mean } \\
\text { of } 5 \text { hours and } 36 \\
\text { minutes training } \\
\text { sessions. }\end{array}$ & $\begin{array}{l}\text { - The French- } \\
\text { Canadian } \\
\text { version of WST }\end{array}$ & $\begin{array}{l}\text { - WSTP training } \\
\text { improves } \\
\text { wheelchair skills } \\
\text { immediately } \\
\text { after training } \\
\text { especially at } \\
\text { community- } \\
\text { skills level. } \\
\text { - The WSTP } \\
\text { group scores }\end{array}$ \\
\hline
\end{tabular}




\begin{tabular}{|c|c|c|c|c|c|c|}
\hline Author & Aim & Study Design & Sample size & $\begin{array}{l}\text { Intervention/ } \\
\text { Module }\end{array}$ & $\begin{array}{l}\text { Outcomes } \\
\text { measure }\end{array}$ & Findings \\
\hline & & & & & & $\begin{array}{l}\text { higher on post- } \\
\text { test. }\end{array}$ \\
\hline $\begin{array}{l}\text { Sawatzky, } \\
\text { Rushton, } \\
\text { Denison, } \\
\text { McDonald } \\
\text { (2012) }\end{array}$ & $\begin{array}{l}\text { - To determine the } \\
\text { effectiveness of a two-day } \\
\text { modified Wheelchair Skills } \\
\text { Programme } 3.2 \text { for children. }\end{array}$ & $\begin{array}{l}\text { - Single group } \\
\text { design. }\end{array}$ & $\begin{array}{l}\text { - Children (ages } \\
\text { 6-19 years) } \\
\text { with SCl or } \\
\text { spina bifida. }\end{array}$ & $\begin{array}{l}\text { - The WSTP } \\
\text { consisted of } 9 \\
\text { hours training } \\
\text { over two days. It } \\
\text { involved the } \\
\text { testing of the } \\
\text { wheelchair skills } \\
\text { (WST) as well as } \\
\text { training } \\
\text { wheelchair skills } \\
\text { like the WSTP }\end{array}$ & $\begin{array}{l}\text { - Wheelchair } \\
\text { Skills Test. }\end{array}$ & $\begin{array}{l}\text { - A two-day } \\
\text { wheelchair skills } \\
\text { programme can } \\
\text { potentially } \\
\text { improve skill } \\
\text { level in children } \\
\text { with spinal cord } \\
\text { injuries or spina } \\
\text { bifida. }\end{array}$ \\
\hline $\begin{array}{l}\text { Sakakibara, } \\
\text { Miller, } \\
\text { Souza, } \\
\text { Nikolova \& } \\
\text { Best (2013) }\end{array}$ & $\begin{array}{l}\text { - To examine the effects of } \\
\text { wheelchair skills training on } \\
\text { confidence in older adults } \\
\text { who are inexperienced with } \\
\text { using a wheelchair }\end{array}$ & $\begin{array}{l}\text { - Single blind } \\
\text { randomized } \\
\text { controlled trial }\end{array}$ & $\begin{array}{l}\text { - } 20 \text { community- } \\
\text { living older } \\
\text { adults }\end{array}$ & $\begin{array}{l}\text { - The intervention } \\
\text { group received } \\
\text { two 1-hour } \\
\text { training sessions } \\
\text { that followed the } \\
\text { Wheelchair Skills } \\
\text { Training Program } \\
\text { (WSTP) protocol. } \\
\text { - The control group } \\
\text { received a single } \\
\text { socialization } \\
\text { contact. }\end{array}$ & $\begin{array}{l}\text { - Wheelchair } \\
\text { Use } \\
\text { Confidence } \\
\text { Scale-Manual } \\
\text { (WheelCon-M) }\end{array}$ & $\begin{array}{l}\text { - WSTP improve } \\
\text { confidence in } \\
\text { using a manual } \\
\text { wheelchair } \\
\text { among } \\
\text { inexperience } \\
\text { older adults. } \\
\text { - The confidence } \\
\text { scores after } \\
\text { post } \\
\text { intervention is } \\
\text { higher. }\end{array}$ \\
\hline $\begin{array}{l}\text { Morgan, } \\
\text { Engsberg, } \\
\text { Gray (2014) }\end{array}$ & $\begin{array}{l}\text { - Identify similarities and } \\
\text { differences between the } \\
\text { perspectives of health care } \\
\text { professionals and manual } \\
\text { wheelchair users and use } \\
\text { the ICF to organize themes } \\
\text { related to rehabilitation and } \\
\text { learning how to use a } \\
\text { manual wheelchair user. }\end{array}$ & $\begin{array}{l}\text { - Descriptive } \\
\text { (Focus Group } \\
\text { to collect } \\
\text { qualitative } \\
\text { data) }\end{array}$ & $\begin{array}{l}\text { - } 13 \text { healthcare } \\
\text { professionals } \\
\text { - } 14 \mathrm{SCl}\end{array}$ & $\begin{array}{l}\text { - Focus group } \\
\text { conducted with } \\
\text { health care } \\
\text { professionals and } \\
\text { wheelchair users } \\
\text { to identify } \\
\text { wheelchair } \\
\text { themes. }\end{array}$ & $\begin{array}{l}\text { - International } \\
\text { Classification } \\
\text { of Functioning, } \\
\text { Disability, and } \\
\text { Health (ICF) }\end{array}$ & $\begin{array}{l}\text { - Identifying } \\
\text { essential } \\
\text { components for } \\
\text { training the } \\
\text { proper } \\
\text { propulsion } \\
\text { mechanics and } \\
\text { wheelchair skills } \\
\text { in new-manual } \\
\text { wheelchair } \\
\text { users is an } \\
\text { essential step in } \\
\text { preventing } \\
\text { future health } \\
\text { and } \\
\text { participation } \\
\text { restrictions. }\end{array}$ \\
\hline $\begin{array}{l}\text { Mountain, } \\
\text { Kirby Smith } \\
\text { Eskes \& } \\
\text { Thompson } \\
\text { (2014) }\end{array}$ & $\begin{array}{l}\text { To test the hypotheses that } \\
\text { people with stroke using } \\
\text { powered wheelchairs who } \\
\text { receive formal wheelchair } \\
\text { skills training improve their } \\
\text { wheelchair skills. }\end{array}$ & $\begin{array}{l}\text { - Randomized } \\
\text { controlled trail. }\end{array}$ & $\begin{array}{l}\text { - } 17 \text { stroke } \\
\text { patients }\end{array}$ & $\begin{array}{l}\text { - Training based on } \\
\text { Wheelchair Skills } \\
\text { Training Program. } \\
\text { - In intervention } \\
\text { group, participants } \\
\text { received } \\
\text { Wheelchair Skills } \\
\text { Training up to } 5 \\
\text { 30-minutes one } \\
\text { on one training } \\
\text { sessions. }\end{array}$ & $\begin{array}{l}\text { - Wheelchair } \\
\text { Skills Test } \\
\text { (WST). }\end{array}$ & $\begin{array}{l}\text { - The change of } \\
\text { WST score in } \\
\text { intervention } \\
\text { group was } \\
\text { notable. } \\
\text { - Wheelchair } \\
\text { skills improve } \\
\text { after wheelchair } \\
\text { skills training. }\end{array}$ \\
\hline $\begin{array}{l}\text { Hashim, Jee, } \\
\text { Lee, Hanapi, } \\
\text { Lee, Fauzi, } \\
\text { Engkasan, } \\
\text { Hasnan } \\
(2016)\end{array}$ & $\begin{array}{l}\text { Determine the effect of an } \\
\text { advanced wheelchair skills } \\
\text { training on wheelchair } \\
\text { mobility function in persons } \\
\text { with Spinal Cord Injury } \\
\text { (SCl). }\end{array}$ & $\begin{array}{l}\text { - A pre and post } \\
\text { intervention } \\
\text { study }\end{array}$ & $\begin{array}{l}\text { - } 19 \text { persons with } \\
\text { traumatic or } \\
\text { non-traumatic } \\
\text { SCI }\end{array}$ & $\begin{array}{l}\text { - Advance } \\
\text { Wheelchair } \\
\text { Training } \\
\text { Workshop } \\
\text { (AWTW). } \\
\text { - Training protocol } \\
\text { was adapted from } \\
\text { Wheelchair Skills } \\
\text { Manual } \\
\text { Programme. } \\
\text { - Consisted of } 12 \\
\text { varying } \\
\text { wheelchair skills } \\
\text { including } \\
\text { propulsion, } \\
\text { performing }\end{array}$ & $\begin{array}{l}\text { - Wheelchair } \\
\text { Mobility Test } \\
\text { (WMT). }\end{array}$ & $\begin{array}{l}\text { - Skills in } \\
\text { ascending stairs } \\
\text { showed most } \\
\text { improvement } \\
\text { followed by } \\
\text { descending } \\
\text { stairs. } \\
\text { - Improvements } \\
\text { in back wheel } \\
\text { balance, } \\
\text { overcoming } \\
\text { small threshold } \\
\text { and low } \\
\text { platform }\end{array}$ \\
\hline
\end{tabular}




\begin{tabular}{|c|c|c|c|c|c|c|}
\hline Author & Aim & Study Design & Sample size & $\begin{array}{l}\text { Intervention/ } \\
\text { Module }\end{array}$ & $\begin{array}{l}\text { Outcomes } \\
\text { measure }\end{array}$ & Findings \\
\hline & & & & $\begin{array}{l}\text { wheelie, } \\
\text { negotiating } \\
\text { platform, } \\
\text { threshold, or } \\
\text { grater, and } \\
\text { managing fall, } \\
\text { ramp, and stairs. }\end{array}$ & & \\
\hline $\begin{array}{l}\text { Kirby, Miller, } \\
\text { Routhier, } \\
\text { Demers, } \\
\text { Mihailidis, } \\
\text { Polgar, } \\
\text { Rushton, } \\
\text { Titus, Smith, } \\
\text { McAllister } \\
\text { (2015) }\end{array}$ & $\begin{array}{l}\text { - To test the hypothesis that } \\
\text { powered wheelchair users } \\
\text { who receive the Wheelchair } \\
\text { Skills Training Program } \\
\text { (WSTP) improve their } \\
\text { wheelchair skills in } \\
\text { comparison with a control } \\
\text { group that receives standard } \\
\text { care. }\end{array}$ & $\begin{array}{l}\text { - Randomized } \\
\text { controlled trial. }\end{array}$ & $\begin{array}{l}\text { - } 116 \text { powered } \\
\text { wheelchair } \\
\text { users. }\end{array}$ & $\begin{array}{l}\text { - Wheelchair Skills } \\
\text { Training Program } \\
\text { (WSTP). } \\
\text { - Five } 30 \text { minutes } \\
\text { WSTP training } \\
\text { sessions for } \\
\text { participants in } \\
\text { WSTP group. }\end{array}$ & $\begin{array}{l}\text { - Wheelchair } \\
\text { Skills Test } \\
\text { Questionnaire } \\
\text { (WST-Q). } \\
\text { - Goal } \\
\text { Attainment } \\
\text { Score (GAS) } \\
\text { - Satisfaction } \\
\text { Questionnaire } \\
\text { - Injury Rate } \\
\text { - Wheelchair } \\
\text { Use } \\
\text { Confidence } \\
\text { Scale for } \\
\text { Power } \\
\text { Wheelchair } \\
\text { Users } \\
\text { (WheelCon) } \\
\text { - Life Space } \\
\text { Assessment. }\end{array}$ & $\begin{array}{l}\text { - Participants in } \\
\text { WSTP group } \\
\text { demonstrate } \\
\text { modest } \\
\text { transient post- } \\
\text { training } \\
\text { improvements } \\
\text { in WST-Q } \\
\text { scores, } \\
\text { performance } \\
\text { scores and they } \\
\text { have substantial } \\
\text { improvements } \\
\text { on } \\
\text { individualized } \\
\text { goals. }\end{array}$ \\
\hline $\begin{array}{l}\text { Kirby, } \\
\text { Mitchell, } \\
\text { Sabharwal, } \\
\text { McCranie \& } \\
\text { Nelson } \\
(2016)\end{array}$ & $\begin{array}{l}\text { - To test hypotheses that } \\
\text { community-dwelling } \\
\text { veterans with spinal cord } \\
\text { injury (SCI) who receive } \\
\text { WSTP in their environments } \\
\text { significantly improve their } \\
\text { manual wheelchair-skills } \\
\text { capacity, retain those } \\
\text { improvements at one year } \\
\text { and improve participation in } \\
\text { comparison with educational } \\
\text { Control (EC) group. }\end{array}$ & $\begin{array}{l}\text { - Randomized } \\
\text { Controlled } \\
\text { Trial }\end{array}$ & $\begin{array}{l}\text { - } 106 \text { veterans } \\
\text { with SCl }\end{array}$ & $\begin{array}{l}\text { - WSTP } \\
\text { - Each participant } \\
\text { receives either } \\
\text { five one to one } \\
\text { WSTP or EC } \\
\text { sessions for } 30-45 \\
\text { minutes. }\end{array}$ & $\begin{array}{l}\text { - Wheelchair } \\
\text { Skills Test } 4.1 \\
\text { (WST). } \\
\text { - Craig Handicap } \\
\text { Assessment } \\
\text { and Reporting } \\
\text { Technique } \\
\text { (CHART) } \\
\text { scores. }\end{array}$ & $\begin{array}{l}\text { - Participants in } \\
\text { WSTP group } \\
\text { improved their } \\
\text { total and } \\
\text { advanced level } \\
\text { WST scores } \\
\text { and retain their } \\
\text { scores at one- } \\
\text { year follow-up. }\end{array}$ \\
\hline $\begin{array}{l}\text { Yeo, Kwon } \\
(2018)\end{array}$ & $\begin{array}{l}\text { - Examine the effectiveness of } \\
\text { wheelchair skills training in } \\
\text { improving both wheelchair } \\
\text { skills and upper arm skilled } \\
\text { performance in adults with } \\
\text { C-SCl. }\end{array}$ & $\begin{array}{l}\text { - Randomized } \\
\text { control Trial }\end{array}$ & - 24 tetraplegia & $\begin{array}{l}\text { - Wheelchair } \\
\text { training session } \\
\text { for the } \\
\text { intervention group } \\
\text { and regular } \\
\text { exercise sessions } \\
\text { for the control } \\
\text { group. } \\
\text { - The intervention } \\
\text { lasted for eight } \\
\text { weeks with a } \\
\text { frequency of three } \\
\text { days (1 hour/day) } \\
\text { per week, } \\
\text { including warm- } \\
\text { ups, training } \\
\text { programs of each } \\
\text { group, and warm - } \\
\text { downs. }\end{array}$ & $\begin{array}{l}\text { - Wheelchair } \\
\text { Skill Test } \\
\text { (WST) } \\
\text { - Van Lieshout } \\
\text { Test (VLT). }\end{array}$ & $\begin{array}{l}\text { - Wheelchair } \\
\text { skills training is } \\
\text { more beneficial } \\
\text { for adults with } \\
\text { C-SCl than } \\
\text { conventional } \\
\text { exercise. }\end{array}$ \\
\hline $\begin{array}{l}\text { Rushton \& } \\
\text { Daoust } \\
\text { (2018) }\end{array}$ & $\begin{array}{l}\text { - To test the hypothesis that } \\
\text { occupational therapy } \\
\text { students who receive } \\
\text { wheelchair skills training } \\
\text { education using a distributed } \\
\text { practice university -course } \\
\text { approach versus a } \\
\text { condensed-practice boot- } \\
\text { camp approach results in } \\
\text { greater improvements post- }\end{array}$ & $\begin{array}{l}\text { - Quasi- } \\
\text { experimental, } \\
\text { non-equivalent } \\
\text { control group } \\
\text { design. }\end{array}$ & $\begin{array}{l}\text { - } 58 \text { occupational } \\
\text { therapy } \\
\text { students. }\end{array}$ & $\begin{array}{l}\text { - For experimental } \\
\text { group, a 15-week, } \\
45 \text { hours } \\
\text { wheelchair } \\
\text { provision course } \\
\text { in which a total of } \\
24 \text { hours was } \\
\text { dedicated to } \\
\text { wheelchair skills } \\
\text { testing and } \\
\text { training education. }\end{array}$ & $\begin{array}{l}\text { - Wheelchair } \\
\text { Skills Test } \\
\text { Questionnaire } \\
\text { (WST-Q) } \\
\text { - Wheelchair } \\
\text { Use } \\
\text { Confidence } \\
\text { Scale for } \\
\text { Manual } \\
\text { Wheelchair }\end{array}$ & $\begin{array}{l}\text { - Occupational } \\
\text { therapy } \\
\text { students who } \\
\text { receive } \\
\text { wheelchair skills } \\
\text { training using } \\
\text { either a } \\
\text { distributed - } \\
\text { practice } \\
\text { university } \\
\text { course or }\end{array}$ \\
\hline
\end{tabular}




\begin{tabular}{|c|c|c|c|c|c|c|}
\hline Author & Aim & Study Design & Sample size & $\begin{array}{l}\text { Intervention/ } \\
\text { Module }\end{array}$ & $\begin{array}{l}\text { Outcomes } \\
\text { measure }\end{array}$ & Findings \\
\hline & $\begin{array}{l}\text { intervention in relevant } \\
\text { outcomes. }\end{array}$ & & & $\begin{array}{l}\text { - For control group, } \\
\text { an } 8 \text { hours } \\
\text { wheelchair skills } \\
\text { training boot- } \\
\text { camp. }\end{array}$ & $\begin{array}{l}\text { User } \\
\text { (WheelCon) } \\
\text { - Self-Efficacy } \\
\text { on Assessing, } \\
\text { Training and } \\
\text { Spotting } \\
\text { wheelchair } \\
\text { skills (SEATS). }\end{array}$ & $\begin{array}{l}\text { condensed- } \\
\text { practice boot- } \\
\text { camp approach } \\
\text { demonstrated } \\
\text { significant post- } \\
\text { training } \\
\text { improvements } \\
\text { in their WST-Q, } \\
\text { WheelCon and } \\
\text { SEATS scores, } \\
\text { but no } \\
\text { significant } \\
\text { differences } \\
\text { were found } \\
\text { between } \\
\text { groups. }\end{array}$ \\
\hline $\begin{array}{l}\text { Park, Jung } \\
\text { (2019) }\end{array}$ & $\begin{array}{l}\text { - To evaluate the modified } \\
\text { WSP for improving } \\
\text { wheelchair skills capacity, } \\
\text { perceived satisfaction, and } \\
\text { performance in daily } \\
\text { activities for hemiplegia } \\
\text { patients in Korea. }\end{array}$ & - Mixed method & $\begin{array}{l}\text { - } 24 \text { hemiplegic } \\
\text { patients }\end{array}$ & $\begin{array}{l}\text { - Modified version } \\
\text { of Wheelchair } \\
\text { Skills Training } \\
\text { Programs. } \\
\text { - Emphasize } \\
\text { wheelchair fitting } \\
\text { for hemiplegic } \\
\text { patients. } \\
\text { - } 30 \text { minutes } \\
\text { intervention for } 6 \\
\text { weeks. }\end{array}$ & $\begin{array}{l}\text { - Wheelchair } \\
\text { Skills Test } \\
\text { used to } \\
\text { measure skills } \\
\text { capacity. } \\
\text { - Canadian } \\
\text { Occupational } \\
\text { Performance } \\
\text { Measure } \\
\text { (COMP) } \\
\text { - Korean version } \\
\text { of the Modified } \\
\text { Barthel Index } \\
\text { (K-MBI). } \\
\text { - User survey on } \\
\text { wheelchair } \\
\text { training } \\
\text { program. }\end{array}$ & $\begin{array}{l}\text { - Significant } \\
\text { improvements } \\
\text { of WST, COPM, } \\
\text { K-MBI scores in } \\
\text { the } \\
\text { experimental } \\
\text { group, the WST } \\
\text { and COPM } \\
\text { score showed } \\
\text { statistically } \\
\text { insignificant } \\
\text { improvement } \\
\text { compared to the } \\
\text { control group. } \\
\text { - Modified WSP } \\
\text { may prove } \\
\text { useful for } \\
\text { hemiplegic } \\
\text { patients who } \\
\text { exhibit low } \\
\text { volition or are } \\
\text { experiencing } \\
\text { wheelchair use } \\
\text { for the first time. }\end{array}$ \\
\hline
\end{tabular}

\subsection{Results and Findings}

The search resulted in a total of 380 journal articles and after inclusion and exclusion criteria are applied, a total of 15 journal articles are found to be relevant in this study. The journal article ranges from the year 2004 to 2019. Of the included papers, 13 studies investigated wheelchair users, one paper investigated wheelchair users and health professionals and two paper were conducted among students. Nine of the papers use randomized controlled trials, none focus group, one pre and post intervention study, one case study, one quasi experimental design and one mixed-methods and one paper use a single group design. All the articles focus on wheelchair skills training. It was interesting to note, that nine of the articles were reported by Kirby and team. The articles were relevant because Kirby and team reported their work on the effectiveness of wheelchair skills training from various aspects, such as from wheelchair users' and care takers' perspectives. The effectiveness of wheelchair skills training can be discussed through personal feedback from participants, observation, and the use of standardized outcome measures, including self-perceived assessment.

\subsection{Participants' Personal Feedback}

Participants' feedback towards the wheelchair skill training in six studies is discussed and indicating wheelchair skills training is practical and effective to implement. Best et al., (2005) have reported that all participants enjoyed and improved their wheelchair skills and confidence after training. Participants also state that training is useful and safe while noted that without training, they would not attempt advanced skills. Coolen et al., (2004) also stated that almost all participants found the training helpful and enjoyable. A study by Kirby et al., (2014) and Kirby et al., (2015), reported that participants had positive perceptions of the training and found the training useful, understandable and would recommend the training to others. Kirby et al., (2016) reported that the training is advantageous and had given them chance to attempt advanced skills that they would never attempt. MacPhee et al., (2004) state that participants' comments on the training were rather positive and they would recommend the training to others. As wheelchair skills training has positive feedback from participants and improved skills, it showed that wheelchair skills training is effective and useful to be implemented. 


\subsection{Observation}

The effectiveness of wheelchair skills training in nine studies was discussed through researchers' observation. A study by Best et al., (2004) reported that during training, participation of family and friends appears to be motivational and trainers were able to provide caregiver advice to improve wheelchair users' skills. Researchers reported that wheelchair skills training improves wheelchair skills (Coolen et al., 2004; Morgan et al., 2017; Park \& Jung, 2019; Routhier et al., 2012; Sawatzky et al., 2012; Yeo \& Kwon, 2018). Yeo \& Kwon, (2019) also reported that wheelchair skills training is more beneficial than conventional exercise. WSTP that have been modified to suit wheelchair users need also be reported for an effective wheelchair training (Park \& Jung, 2019; Yeo \& Kwon, 2018). Morgan, Engsberg, et al., (2017) also state that learning wheelchair skills would prevent future health and participations restrictions and new wheelchair users need wheelchair skills training that include propulsion techniques, transfer in and out of a wheelchair, providing skills for wheelchair maintenance and wheelchair navigation skill to overcome barriers such as curbs, ramps and rough terrain. Wheelchair skill training improved performance skills in self-care, productivity, and satisfaction (Park \& Jung, 2019) and improved caregivers handling skills and their wheelchair skills (Kirby et al., 2010). These papers suggest that wheelchair skill training does improve wheelchair skills and wheelchair users also need to have the knowledge on their wheelchair to improve their skills.

\subsection{Discussion}

This scoping review is a part of a bigger project on wheelchair modification and training for PWDs. This scoping review was aimed to identify the types of available evidence and analyze knowledge gaps on wheelchair skills training among PWDs in literature.

The available evidence would provide a strong foundation towards developing a wheelchair training program and services.

This review found that wheelchair skills training is more effective than the standard available treatment. Wheelchair skill training was effective in improving health, participations, skills, performance, self-care, productivity, and satisfaction of wheelchair users. Wheelchair skill training is also effective in improving caregivers handling skills. The variability of tests used by the research included in this study shows that wheelchair skills training can also improve wheelchair user confidence, performance, independence and productivity in daily activities, and mobility of wheelchair users. This study also highlights that wheelchair skill training can improve safety awareness and safe practice of using wheelchairs not only for wheelchair users but also for health personnel and caregivers. It is important for wheelchair users to understand the knowledge on wheelchairs and be proficient in wheelchair skills training. Thus, this study highlights the importance and beneficiaries of wheelchair skills training to wheelchair users in Malaysia and provides strong evidence of the needs of wheelchair skills training and the contents of the wheelchair skill training program.

\subsection{Conclusion and Recommendations}

This study identified the types of available evidence and analyzed the knowledge gap on the effectiveness of Wheelchair Skills Training through personal feedbacks from participants, observation and the use of standardized outcome measures, including self-perceived assessment. This scoping review research found that wheelchair skills training is effective in improving wheelchair skills of wheelchair users and caregivers. The review shows that wheelchair skills training is effective to improve wheelchair skills, performance and safety. Therefore, it is relevant to suggest the need to develop a structured wheelchair training program in Malaysia for PWD. This study may assist health personnel in Malaysia, especially to the occupational therapists to apply a specific wheelchair skills training program during the active rehabilitation process. As this study used scoping review method, it is not as rigorously and comprehensive, therefore a more thorough study can be done through a systematic review. Nevertheless, these findings may provide a strong foundation for future research on wheelchair skills training in Malaysia as well as on the effectiveness of wheelchair skills training in Malaysia.

\section{Acknowledgement}

This study is funded by MRUN Translational Grant (USM-MTUN/MCUM) Grant number 304.PPSK.656208. We would also express our gratitude to all staff at Disability Transformation Unit, Universiti Sains Malaysia (USM).

\section{Paper Contribution to Related Field of Study}

The findings of this study suggest the need to develop a structured wheelchair training program in Malaysia for PWD. This study may help health personnel in Malaysia to apply a specific wheelchair skills training program during the active rehabilitation process.

\section{References}

Best, K. L., Routhier, F., \& Miller, W. C. (2015). A description of manual wheelchair skills training: current practices in Canadian rehabilitation centers. Disability and Rehabilitation: Assistive Technology, 10(5), 393-400

Best, K. L., Kirby, R. L., Smith, C., \& MacLeod, D. A. (2005). Wheelchair skills training for community-based manual wheelchair users: a randomized controlled trial. Archives of physical medicine and rehabilitation, 86(12), 2316-2323.leving 
Boninger, M. L., Koontz, A. M., Sisto, S. A., Dyson-Hudson, T. A., Chang, M., Price, R., \& Cooper, R. A. (2005). Pushrim biomechanics and injury prevention in spinal cord injury: recommendations based on CULP-SCl investigations. Journal of rehabilitation research and development, 42(3), 9.

Coolen, A. L., Kirby, R. L., Landry, J., MacPhee, A. H., Dupuis, D., Smith, C., Best, K. L., MacKenzie, D. E., \& MacLeod, D. A. (2004). Wheelchair skills training program for clinicians: A randomized controlled trial with occupational therapy students. Archives of Physical Medicine and Rehabilitation, 85(7), $1160-1167$. https://doi.org/10.1016/j.apmr.2003.10.019

Desai, S., Mantha, S., \& Phalle, V. (2018). Design of a reconfigurable wheelchair with stand-sit-sleep capabilities for enhanced independence of long-term wheelchair users. Technology and Disability, 30(3), 135-151.

Forslund, E. B., Jørgensen, V., Franzén, E., Opheim, A., Seiger, Å., Ståhle, A., Hultling, C., Stanghelle, J.K., Roaldsen K. S., \& Wahman, K. (2017). High incidence of falls and fall-related injuries in wheelchair users with spinal cord injury: a prospective study of risk indicators. Journal of rehabilitation medicine, 49(2), 144-151.

Kirby, R. L., Mifflen, N. J., Thibault, D. L., Smith, C., Best, K. L., Thompson, K. J., \& MacLeod, D. A. (2004). The manual wheelchair-handling skills of caregivers and the effect of training. Archives of Physical Medicine and Rehabilitation, 85(12), 2011-2019. https://doi.org/10.1016/j.apmr.2004.02.014

Kirby, R.L., Rushton, P.W., Smith, C., Routhier, F., Best K.L., Boyce, J., Cowan, R., Giesbrecht, E., Kenyon L.K., Koontz, A., MacKenzie, D., Mortenson, B., Parker, K., Russell, K.F.J., Smith, E., Sonenblum, S., Tawashy, A., Toro M., \& Worobey, L. Wheelchair Skills Program Manual Version 5.0 (2019). Published electronically at Dalhousie University, Halifax, Nova Scotia, Canada. www.wheelchairskillsprogram.ca/eng/manual.php

Kirby, R. L., Mitchell, D., Sabharwal, S., McCranie, M., \& Nelson, A. L. (2016). Manual wheelchair skills training for community-dwelling veterans with spinal cord injury: a randomized controlled trial. PloS one, 11(12), e0168330.

Kirby, R. L., Rushton, P. W., Routhier, F., Demers, L., Titus, L., Miller-Polgar, J., ... \& McAllister, M. (2017). The extent to which caregivers enhance the wheelchair skills of powered wheelchair users: A cross-sectional study. In RESNA ANNUAL CONFERENCE.

Kirby, R. L., Smith, C., Cowen, R., Dyson-Hudson, T., Shea, M., Presperin, J., Oyster, M. \& Boninger, M. (2014, June). A one-day practical workshop can improve the knowledge and skills of therapists and research personnel about the Wheelchair Skills Program. In Proceedings of the Annual Meeting of RESNA.

Kirby, R. L., Swuste, J., Dupuis, D. J., MacLeod, D. A., \& Monroe, R. (2002). The Wheelchair Skills Test: a pilot study of a new outcome measure. Archives of Physical Medicine and Rehabilitation, 83(1), 10-18.

Leving, M. T., Vegter, R. J., de Vries, W. H., de Groot, S., \& van der Woude, L. H. (2018). Changes in propulsion technique and shoulder complex loading following lowintensity wheelchair practice in novices. PloS one, 13(11), e0207291.

Mountain, A. D., Kirby, R. L., Smith, C., Eskes, G., \& Thompson, K. (2014). Powered wheelchair skills training for persons with stroke: a randomized controlled trial. American journal of physical medicine \& rehabilitation, 93(12), 1031-1043.

Park, J. M., \& Jung, H. S. (2019). A study on the effects of modified wheelchair skills program (WSP) for hemiplegic clients. Assistive Technology, 1-8.

Phang, S. H., Martin Ginis, K. A., Routhier, F., \& Lemay, V. (2012). The role of self-efficacy in the wheelchair skills-physical activity relationship among manual wheelchair users with spinal cord injury. Disability and rehabilitation, 34(8), 625-632.

Routhier, F., Kirby, R. L., Demers, L., Depa, M., \& Thompson, K. (2012). Efficacy and retention of the French-Canadian version of the Wheelchair Skills Training Program for manual wheelchair users: a randomized controlled trial. Archives of physical medicine and rehabilitation, 93(6), 940-948.

Sakakibara, B. M., Miller, W. C., Souza, M., Nikolova, V., \& Best, K. L. (2013). Wheelchair skills training to improve confidence with using a manual wheelchair among older adults: a pilot study. Archives of physical medicine and rehabilitation, 94(6), 1031-1037. https://doi.org/10.1016/j.apmr.2013.01.016

Sawatzky, B., Rushton, P. W., Denison, I., \& McDonald, R. (2012). Wheelchair skills training programme for children: a pilot study. Australian occupational therapy journal, $59(1), 2-9$.

Sucharew, H., \& Macaluso, M. (2019). Methods for research evidence synthesis: The scoping review approach. In Journal of Hospital Medicine. https://doi.org/10.12788/jhm.3248

Sumner, E., O'Connell, C., \& Macalpine, B. (2017). Wheelchair donation in a low-resource setting: utilization, challenges and benefits of wheelchairs provided through a specialized seating programme in Haiti. Journal of rehabilitation medicine, 49(2), 178-184.

World Health Organization. (2012). Wheelchair service training package: basic level. World Health Organization https://www.who.int/disabilities/technology/wheelchairpackage/en/. Retrieved on 02/12/2019.

Xiang, H., Chany, A. M., \& Smith, G. A. (2006). Wheelchair related injuries treated in US emergency departments. Injury prevention, 12(1), 8-11.

Yeo, S. S., \& Kwon, J. W. (2018). Wheelchair Skills Training for Functional Activity in Adults with Cervical Spinal Cord Injury. International journal of sports medicine, 39(12), 924-928 